\title{
Informelles Lernen im Kindes- und Jugendalter
}

\author{
Doris Lewalter \& Katrin Neubauer \\ Technische Universität München
}

Für ein umfassendes Verständnis von Bildungsprozessen rückt neben dem formalen Lernen zunehmend auch das informelle Lernen in den Fokus der empirischen Bildungsforschung (Harring, Witte \& Burger, 2016; Hungerland \& Overwien, 2004; Täubig, 2018). Diese Entwicklung spiegelt sich auch in den Beiträgen der Handbücher zum informellen Lernen, die in den letzten Jahren erschienen sind, wider (z. B. Harring et al., 2016; Kahnwald \& Täubig, 2018; Rohs, 2016).

Trotz der zunehmenden wissenschaftlichen Beachtung fehlt bisher eine allgemein akzeptierte Definition des informellen Lernens. Dieses kann im Alltag, im Familienkreis, in der Freizeit oder am Arbeitsplatz stattfinden (Lewalter \& Neubauer, 2019; Rohs, 2016) und wird bislang überwiegend durch Abgrenzungen und Gegenüberstellungen zu anderen Lernformen definiert (z. B. Dohmen, 2016; Düx \& Rauschenbach, 2010; Europäische Kommission, 2001; Lewalter \& Neubauer, 2019). Allgemein wird dabei im Rahmen diskret-gestufter Ansätze (Rohs, 2016) zwischen formalem, non- bzw. nicht-formalem und informellem Lernen unterschieden (vgl. BMFSFJ, 2005; Dohmen, 2001, 2002; Europäische Kommission, 2001; Harring et al., 2016). Die Europäische Kommission (2001) hat diese Lernformen wie folgt definiert: Der Begriff formales Lernen beschreibt Lernen, das üblicherweise in einer formalen Bildungsoder Ausbildungseinrichtung, wie Kindergarten, Schule, Hochschule oder Berufsausbildungseinrichtungen, stattfindet. Diese Lernform ist in Bezug auf Lernziele, Lernzeit oder Lernförderung strukturiert und führt zu einer Zertifizierung. Formales Lernen ist aus der Sicht des Lernenden zielgerichtet und bewusst. Demgegenüber umfasst non- bzw. nicht-formales Lernen Lernprozesse, die nicht in formalen Bildungs- oder Berufsbildungseinrichtungen, aber an klar definierten Orten (z. B. VHS) stattfinden und üblicherweise nicht zur Zertifizierung führen. Gleichwohl ist es systematisch in Bezug auf Lernziele, Lerndauer und Lernmittel und aus Sicht der Lernenden zielgerichtet und bewusst. Mit dem Begriff des informellen Lernens wird das Lernen jenseits formaler Bildungsinstitutionen im Alltag in nicht-inszenierten Settings, z. B. am Arbeitsplatz, Freundeskreis oder in der Freizeit, angesprochen. Es ist in Bezug auf Lernziele, Lernzeit oder Lernförderung nicht strukturiert und führt zu keiner Zertifizierung. Informelles Lernen kann zielgerichtet sein, ist jedoch in den meisten Fällen nichtintentional und erfolgt mitunter auch beiläufig. Die in dieser Definition bereits anklingenden Schwierigkeiten bei der klaren Abgrenzung der einzelnen Lernformen führten zu weiteren Ansätzen der Begriffsbestimmung. So geht zum Beispiel Rohs $(2008,2014)$ von einem Kontinuum zwischen formalem und informellem Lernen aus, in welchem keine klare Trennung der Lernformen vorgesehen ist. Rohs (2008) definiert die Endpunkte dieses Kontinuums von formalem Lernen zu informellem Lernen anhand der Dimensionen Intention, Gegenstand, Steuerung (in Bezug auf Ziele, Inhalte, Zeit), Lernunterstützung, Lernergebnis und Bewusstheit. Formales Lernen folgt demnach einer klaren Lernintention, ist fokussiert und erfolgt fremdgesteuert im Rahmen eines organisierten pädagogischen Angebots, wobei überwiegend Theoriewissen durch bewusste Lernprozesse erworben wird. Demgegenüber ist informelles Lernen überwiegend problemlöseorientiert, wobei der Gegenstand ganzheitlich betrachtet wird. Die Steuerung von Lernziel, -zeit und -inhalt erfolgt selbstbestimmt ohne organisierte Lernunterstützung, die lediglich auf Nachfrage erfolgt. 
Das Lernergebnis bezieht sich überwiegend auf Erfahrungswissen. Der informelle Lernprozess kann in Teilen auch unbewusst ablaufen.

Zur Charakterisierung der Beziehung zwischen informellem Lernen und formalem Lernen werden in der Literatur häufig die Begriffe Integration und Verbindung verwendet (s. zsfd. Rohs, 2014). In beiden Fällen wird von der Annahme ausgegangen, dass Bezüge zwischen beiden Lernformen vorhanden sind bzw. hergestellt werden können. Der Begriff Integration bezieht sich meist auf den Einbezug des informellen Lernens in das formale Lernen, wobei die Gefahr besteht, dass dabei die spezifischen Charakteristika des informellen Lernens verloren gehen. Mit dem Begriff Verbindung wird die Kombination bzw. das gleichberechtigte Zusammenwirken beider Lernformen unter Beibehaltung ihrer je spezifischen Eigenheiten beschrieben. Entsprechend wird, bezogen auf unterschiedliche Bildungskontexte, wiederholt betont, dass informelles und formales Lernen nicht als Gegensatzpaar betrachtet werden sollten, sondern vielmehr als sich wechselseitig ergänzende und befruchtende Lernformen (vgl. Europäische Kommission, 2001; Harring et al., 2016). Rauschenbach (2016) stellt für den schulischen Kontext fest, dass sich hier informelles und formales Lernen wechselseitig beeinflussen können. Wie dieses Zusammenwirken im Detail aussehen kann, hängt u. a. von zeitlichen, inhaltlichen und kontextuellen Rahmenbedingungen ab (Rohs, 2014). Damit werden die zeitliche Abfolge der Lernformen, deren inhaltliche Verbindung oder Koppelung sowie die zufälligen oder gezielten Bezüge der jeweiligen formalen bzw. informellen Lernkontexte zueinander angesprochen.

Bezogen auf das Zusammenwirken von formalem und informellem Lernen im Kindesund Jugendalter ist davon auszugehen, dass das informelle Lernen, das teilweise vorgelagert und zeitgleich zum formalen schulischen Lernen stattfindet, schulisches Lernen vorbereiten, unterstützen und ergänzen, aber auch von diesem profitieren kann. Des Weiteren ist zu beachten, dass auch in der Schule nicht nur formal orga- nisiertes Lernen, sondern auch non-formales und informelles Lernen, zum Beispiel in den Pausen oder während Ausflügen sowie Angeboten im Ganztagsbereich oder während PeerLearning-Situationen stattfinden können, die sich u. a. auf das Erlernen sozialer Regeln sowie die Ausbildung der sozialen Identität beziehen (Coelen, Gusinde, Lieske \& Trautmann, 2016; Rohlfs \& Hertel, 2016). Rohs betont, dass das Zusammenwirken formalen und informellen Lernens „in keine Richtung determiniert ist, sondern als kontinuierlicher Prozess des wechselseitigen Aufeinanderfolgens verstanden werden kann, der spiralförmig zu einer Steigerung des Wissens- und Kompetenzniveaus führt" (Rohs, 2014, S. 399).

Die inhaltliche Verbindung beider Lernformen stellt eine große (didaktische) Herausforderung dar. Denn informelles Lernen im Kindesund Jugendalter findet insbesondere in der Familie, mit Peers oder Medien sowie in Vereinen statt (Düx \& Rauschenbach, 2016; Lewalter \& Neubauer, 2019) und entzieht sich damit weitüberwiegend einer externen Steuerung (Rohs, 2014). Der Familie kommt eine sowohl vorbereitende als auch begleitende Aufgabe für das formale Lernen im schulischen Kontext zu. So werden zum Beispiel für den schulischen Lernerfolg wesentliche Fertigkeiten und Kenntnisse, wie die Sprache oder der überlegte und differenzierende Umgang mit Informationen, zum Teil bereits vorgelagert im informellen familiären Kontext erworben oder die im privaten Umfeld erworbenen sozialen Kompetenzen im Schulkontext genutzt. Peers nehmen sowohl in der Schule als auch in der Freizeit Einfluss auf formale ebenso wie informelle Lernprozesse, beispielsweise im Rahmen der Identitätsentwicklung (Düx \& Rauschenbach, 2016; Lewalter \& Neubauer, 2019). Die Nutzung von Medien in der Freizeit stellt einen weiteren Kontext informellen Lernens dar, der aufgrund der dadurch erworbenen Medienkompetenz bzw. Medienbildung (s. zsfd. Lewalter \& Neubauer, 2019; Pietraß, 2016) auch für das formale Lernen in der Schule von zentraler Bedeutung ist (Düx \& Rauschenbach, 2016). 
In der aktuellen Diskussion geht es vor allem darum, Wege zu finden, wie informelles, nonformales und formales Lernen sinnvoll und in ergänzender Weise miteinander verknüpft werden können (Eshach, 2007). Dazu ist es u. a. wichtig, die Kenntnisse, die non-formal oder informell innerhalb oder außerhalb der Schule erworben wurden, stärker als bisher in der formalen schulischen Ausbildung aufzugreifen und gezielt einzubeziehen. Hierbei bestehen zahlreiche offene Fragen, die sich u. a. auf die Gestaltung wechselseitig förderlicher Rahmenbedingungen für deren effektives Zusammenspiel beziehen. Dies wird beispielsweise im Ansatz der sogenannten peer education (Heyer, 2010) versucht, der davon ausgeht, dass Peers aufgrund ihres Einflusses auf Gleichaltrige als Bezugs- und Orientierungspunkte dienen können, die Lernund Bildungsprozesse unterstützen können.

Diese Neubewertung des schulischen und informellen Kompetenzerwerbs geht mit einer Veränderung des Rollenverständnisses von Lehrenden und Lernenden und einer Abkehr vom wissensdominierten hin zum kompetenzentwickelnden Lernen einher (vgl. Delors, 1996; OECD, 1996; Overwien, 2009). Nach Hungerland und Overwien (2004) bedarf es der Etablierung einer partizipativen schulischen Lernkultur, in welcher die Lernenden aufgefordert werden, ihre außerschulisch erworbenen Kompetenzen in den Unterricht aktiv einzubringen, und in welcher Lehrende als professionelle Lernbegleiter fungieren, die eigenständige Lernprozesse der Lernenden anregen und begleiten.

Eine schwierige Aufgabe im Zusammenhang mit der veränderten Sichtweise auf das informelle und formale Lernen stellt dabei die Erforschung des informellen Lernens dar, welche die empirische Bildungsforschung vor große Herausforderungen stellt. Diese basieren u. a. auf der Komplexität und der uneinheitlichen Definitionslage (Böhm-Kasper \& Bienefeld, 2016) sowie den teilweise unbewusst und beiläufig ablaufenden Lernprozessen des informellen Lernens und der geringen Zugänglichkeit lebensweltlicher informeller Lernkontexte wie Familie oder Peers (Düx \& Rauschenbach, 2016). Spe- zifische Ausschnitte des informellen Lernens werden derzeit mit sehr unterschiedlichen methodischen Zugängen in den Blick genommen. Diese methodischen Zugänge reichen von qualitativer und quantitativer Bildungsforschung über ethnografische Ansätze, dokumentarische Methoden und Biografieforschung bis hin zu Evaluationsforschung (s. Beiträge in Harring et al., 2016).

\section{Literatur}

BMFSFJ. (Hrsg.). (2005). Zwölfter Kinder- und Jugendbericht. Bericht über die Lebenssituation junger Menschen und die Leistung der Kinder- und Jugendhilfe in Deutschland. Zwölfter Kinder-und Jugendbericht Bildung, Betreuung und Erziehung vor und neben der Schule. Bonn: Bundesministerium für Familie, Senioren, Frauen und Jugend.

Böhm-Kasper, O. \& Bienefeld, M. (2016). Quantitative Bildungsforschung und informelles Lernen. In $M$. Harring, M. D. Witte \& T. Burger (Hrsg.), Handbuch informelles Lernen. Interdisziplinäre und internationale Perspektiven (S. 710-726). Weinheim: Beltz Juventa.

Coelen, T., Gusinde, F., Lieske, N. \& Trautmann M. (2016). Informelles Lernen in der Schule. In M. Rohs (Hrsg.), Handbuch Informelles Lernen (S. 325-342). Wiesbaden: Springer. https://dx.doi.org/10.1007/9783-658-05953-8_39

Delors, J. (1996). Learning. The treasure within. Paris: UNESCO Publishing.

Dohmen, G. (2001). Das informelle Lernen. Die internationale Erschließung einer bisher vernachlässigten Grundform menschlichen Lernens für das lebenslange Lernen aller. Bonn: Bundesministerium für Bildung und Forschung.

Dohmen, G. (2002). Informelles Lernen in der Freizeit. Spektrum Freizeit, 24(1), 18-27.

Dohmen, G. (2016). Das informelle Lernen. In M. Harring, M. D. Witte \& T. Burger (Hrsg.), Handbuch informelles Lernen. Interdisziplinäre und internationale Perspektiven (S. 53-62). Weinheim: Beltz Juventa.

Düx, W. \& Rauschenbach, T. (2010). Informelles Lernen im Jugendalter. In N. Neuber (Hrsg.), Informelles Lernen im Sport. Beiträge zur allgemeinen Bildungsdebatte (S. 53-77). Wiesbaden: VS Verlag für Sozialwissenschaften. https://dx.doi.org/10.1007/978-3-531-9243 9-7_3

Düx, W. \& Rauschenbach, T. (2016). Informelles Lernen im Jugendalter. In M. Rohs (Hrsg.), Handbuch informelles Lernen (S. 261-283). Wiesbaden: Springer Verlag. https://dx.doi.org/10.1007/978-3-658-05953-8_18

Eshach, H. (2007). Bridging in school and out of school learning: Formal, non-formal and informal education. Journal of Science Education and Technology, 16, 171 190. https://dx.doi.org/10.1007/s10956-006-9027-1

Europäische Kommission, Generaldirektion Bildung und Kultur, Generaldirektion Beschäftigung und Soziales. Mitteilung der Kommission (2001). Einen europäischen Raum des Lebenslangen Lernens schaffen. Luxembourg: Europäische Kommission. 
Harring, M., Witte, M. D. \& Burger, T. (2016). Handbuch informelles Lernen. Interdisziplinäre und internationale Perspektiven. Weinheim: Beltz Juventa.

Heyer, R. (2010). Peer-Education Ziele, Möglichkeiten, Grenzen. In M. Harring, O. Böhm-Kasper, C. Rohlfs \& C. Palentien (Hrsg.), Freundschaften, Cliquen und Jugendkulturen. Peers als Bildungs- und Sozialisationsinstanz (S. 407-421). Wiesbaden: VS Verlag für Sozialwissenschaften. https://dx.doi.org/10.1007/978-3531-92315-4_19

Hungerland, B. \& Overwien, B. (2004). Kompetenzerwerb außerhalb etablierter Lernstrukturen. In B. Hungerland \& B. Overwien (Hrsg.), Kompetenzentwicklung im Wandel. Auf dem Weg zu einer informellen Lernkultur? (S. 1-23). Wiesbaden: VS Verlag für Sozialwissenschaften. https://dx.doi.org/10.1007/978-3-531-901 62-6_1

Kahnwald, N. \& Täubig, V. (2018). Informelles Lernen: Standortbestimmungen. Wiesbaden: Springer. https:// dx.doi.org/10.1007/978-3-658-15793-7

Lewalter D. \& Neubauer K. (2019). Informelles Lernen. In D. Urhahne, M. Dresel, F. Fischer (Hrsg.), Psychologie für den Lehrberuf(S. 125-142). Berlin: Springer. https://dx.doi.org/10.1007/978-3-662-55754$9+7$

OECD. (1996). Lifelong learning for all. Meeting of the education committee at ministerial level. 16. - 17. Januar, Paris.

Overwien, B. (2009). Informelles Lernen. Definitionen und Forschungsansätze. In M. Brodowski, U. Devers-Kanoglu, B. Overwien, M. Rohs, S. Salinger \& M. Walser (Hrsg.), Informelles Lernen und Bildung für eine nachhaltige Entwicklung. Beiträge aus Theorie und Praxis (S 23-34). Farmington Hills, MI: Verlag Barbara Budrich. https://dx.doi.org/10.2307/j.ctvddzjqt.5

Pietraß, M. (2016). Informelles Lernen in der Medienpädagogik. In M. Rohs (Hrsg.), Handbuch informelles Lernen (S. 123-142). Wiesbaden: Springer. https://dx doi.org/10.1007/978-3-658-05953-8_10
Rauschenbach, T. (2016). Informelles Lernen Bilanz und Perspektiven. In M. Harring, M. D. Witte \& T. Burger (Hrsg.), Handbuch informelles Lernen. Interdisziplinäre und internationale Perspektiven (S. 803-817). Weinheim: Beltz Juventa.

Rohlfs, C. \& Hertel, S. (2016). Informelles Lernen in Schule und Unterricht. In M. Harring, M. D. Witte \& T. Burger (Hrsg.), Handbuch informelles Lernen. Interdisziplinäre und internationale Perspektiven (S. 633-646). Weinheim: Beltz Juventa.

Rohs, M. (2008). Connected learning: Zur Verbindung formellen und informellen Lernens in der IT-Weiterbildung. VDM Publishing.

Rohs, M. (2014). Konzeptioneller Rahmen zum Verhältnis formellen und informellen Lernens. Swiss Journal of Educational Science, 36, 391-406.

Rohs, M. (2016). Genese informellen Lernens. In M. Rohs (Hrsg.), Handbuch informelles Lernen (S. 3-38). Wiesbaden: Springer Verlag. https://dx.doi.org/10.1007/ 978-3-658-05953-8_1

Täubig, V. (2018). Informelles Lernen. In N. Kahnwald \& V. Täubig (Hrsg.), Informelles Lernen. Standortbestimmungen. Wiesbaden: Springer. https://dx.doi.org/10. 1007/978-3-658-15793-7__

\section{Prof. Dr. Doris Lewalter}

Technische Universität München

School of Education

Arcisstr. 21, 80333 München

E-Mail: doris.lewalter@tum.de

\section{Dr. Katrin Neubauer}

Technische Universität München

School of Education

Arcisstr. 21, 80333 München

E-Mail: katrin.neubauer@tum.de 